\title{
FGFR2 Mutation Confers a Less Drastic Gain of Function in Mesenchymal Stem Cells Than in Fibroblasts
}

\author{
Erika Yeh • Rodrigo Atique • Felipe A. A. Ishiy • Roberto Dalto Fanganiello • \\ Nivaldo Alonso - Hamilton Matushita - Katia Maria da Rocha • \\ Maria Rita Passos-Bueno
}

Published online: 3 November 2011

(C) The Author(s) 2011. This article is published with open access at Springerlink.com

\begin{abstract}
Gain-of-function mutations in FGFR2 cause Apert syndrome (AS), a disease characterized by craniosynostosis and limb bone defects both due to abnormalities in bone differentiation and remodeling. Although the periosteum is an important cell source for bone remodeling, its role in craniosynostosis remains poorly characterized. We hypothesized that periosteal mesenchymal stem cells (MSCs) and fibroblasts from AS patients have abnormal cell phenotypes that contribute to the recurrent fusion of the coronal sutures. MSCs and fibroblasts were obtained from the periostea of 3 AS patients (S252W) and 3 control individuals (WT). We evaluated the proliferation, migration, and osteogenic differentiation of these cells. Interestingly, S252W mutation had opposite effects on different cell types: S252W MSCs proliferated less than WT MSCs, while S252W fibroblasts proliferated more than WT fibroblasts. Under restrictive media conditions, only S252W fibroblasts showed enhanced migration. The presence of $\mathrm{S} 252 \mathrm{~W}$ mutation increased in vitro and in vivo osteogenic differentiation in both studied cell types, though the difference compared to WT cells was more pronounced in S252W fibroblasts. This osteogenic
\end{abstract}

Electronic supplementary material The online version of this article (doi:10.1007/s12015-011-9327-6) contains supplementary material, which is available to authorized users.

E. Yeh $\cdot$ R. Atique $\cdot$ F. A. A. Ishiy $\cdot$ R. D. Fanganiello

K. M. da Rocha $\cdot$ M. R. Passos-Bueno $(\square)$

Human Genome Center, Department of Genetics and Evolutive

Biology, Institute of Bioscience, University of Sao Paulo,

Rua do Matão, 277,

São Paulo, SP CEP 05508-900, Brazil

e-mail: passos@ib.usp.br

N. Alonso $\cdot$ H. Matushita

Department of Plastic Surgery, School of Medicine,

University of Sao Paulo,

Sao Paulo, Brazil differentiation was reversed through inhibition of JNK. We demonstrated that $\mathrm{S} 252 \mathrm{~W}$ fibroblasts can induce osteogenic differentiation in periosteal MSCs but not in MSCs from another tissue. MSCs and fibroblasts responded differently to the pathogenic effects of the FGFR2 ${ }^{\mathrm{S} 252 \mathrm{~W}}$ mutation. We propose that cells from the periosteum have a more important role in the premature fusion of cranial sutures than previously thought and that molecules in JNK pathway are strong candidates for the treatment of AS patients.

Keywords Mesenchymal stem cells · Fibroblasts · FGFR2 . Apert syndrome $\cdot$ Osteogenesis

\section{Introduction}

Apert Syndrome (AS), the most severe form of craniosynostosis [1], is characterized by premature fusion of the coronal sutures, severe syndactyly of the hands and feet and by a range of skeletal abnormalities, including progressive joint limitation [2-4]. This autosomal dominant syndrome is caused by gain-of-function mutations in the FGFR2 gene. The most prevalent mutation, accounting for approximately $65 \%$ of all AS cases, is S252W. FGFR2, by means of alternative mRNA splicing, can be transcribed into an epithelial and a mesenchymal isoform. Both isoforms are tyrosine kinase receptors that bind to a specific subset of fibroblast growth factors (FGFs) to induce a variety of cell functions, such as cell migration, proliferation, and differentiation [5]. In the presence of the S252W mutation, FGFR2 shows enhanced ligand-binding affinity to FGF2 and loses isoform-ligand specificity for most of the other ligands [6]. This mutation affects both the epithelial and the mesenchymal FGFR2 isoforms. Although most of the clinical features of AS arise as a consequence of signaling disturbance during 
embryonic development, FGFR2 $2^{\mathrm{S} 252 \mathrm{~W}}$ also interferes in postnatal organism homeostasis. Surgical opening of the coronal sutures is a mandatory procedure for AS patients. However, the excessive and repetitive closure of these sutures after the procedure (resynostosis) requires multiple interventions from birth until adulthood [1].

It has been postulated that $\mathrm{FGFR} 2^{\mathrm{S} 252 \mathrm{~W}}$ enhances cell proliferation, which would account for the higher ossification rate at the sutures [7]. However, we still do not know if this enhanced proliferation is observed in all cell types. Most studies have focused on murine osteoblasts harboring the AS mutation, but both increased [8] and decreased [9] proliferation have been observed. Studies on the effect of FGFR $2^{\mathrm{S} 252 \mathrm{~W}}$ on osteogenic potential have also produced contradictory results [8-10]. Few studies have investigated the functional effect of FGFR2 ${ }^{\mathrm{S} 252 \mathrm{~W}}$ in human cells, which are considerably different from murine cells in regard to cell signaling [11-16]. We have conducted a preliminary study in which we suggested that S252W fibroblasts have an increased osteogenic potential [17], a finding that we sought to replicate using in vivo models. On the other hand, there are no studies on human AS mesenchymal stem cells (MSC), the possible precursors of osteoblasts. The premature suture fusion and the resynostosis process after surgical interventions are not likely the result of alterations in one particular cell type, such as osteoblasts, but the result of perturbations in signaling and in interactions between different cell types and tissues of the cranial suture complex [18].

The cranial suture complex comprises the overlying periosteum of the calvaria, the osteogenic fronts of the bone plates, the intervening mesenchyme, and the underlying dura mater. This complex allows skull deformation during birth, expansion during brain growth and regulates the balance between proliferation and differentiation of osteogenic precursors [18]. Several studies have indicated dura mater as a crucial tissue that regulates suture patency, so it is assumed that signaling by this tissue is deregulated in AS patients, contributing to premature embryonic synostosis as well as adult resynostosis $[1,19]$. Remarkably, the removal of the periosteum diminishes calcification of cranial defects in animal models [20, 21], which highlights the importance of the periosteum in cranial bone formation. The periosteum contributes not only to normal bone growth, but also to bone healing and regeneration [22, 23]. This highly cellular tissue contains multipotent MSC, fibroblasts, differentiated osteogenic progenitor cells and osteoblasts $[24,25]$, and acts as a major source of skeletal stem cells/ progenitors during bone repair [26]. It is thus possible that periosteal cells also have a major contribution to the premature suture fusion in AS patients; however, their role in this process is still poorly characterized. In addition, it is still unknown how the different cell types from the calvarial periosteum interact and what is the functional effect of the FGFR2 $2^{\mathrm{S} 252 \mathrm{~W}}$ mutation in these cells.

JNK (c-Jun N-terminal kinases) has been reported as crucial for the final stage of differentiation in preosteoblasts and pluripotent cells [27-30], and is considered a critical regulator of important osteogenic differentiation markers [30, 31]. In addition, two negative regulators of JNK activity of the same family are associated with craniosynostosis: DUSP6 and DUSP2. The loss of Dusp6 leads to coronal craniosynostosis in mice [32], and we have previously reported that DUSP2 was one of the most significant differentially expressed genes in AS periosteal cells [17]. Therefore, JNK is an interesting candidate for the altered osteogenic potential of S252W cells.

In view of the above, we conducted this study to access the effect of the FGFR2 ${ }^{\mathrm{S} 252 \mathrm{~W}}$ mutation in fibroblasts and MSCs derived from the coronal periosteum. We examined these cells' proliferative capacity, motility, and osteogenic potential and also evaluated whether there is a functional interaction between them. Finally, we have evaluated the role of JNK in the increased osteogenic potential of S252W fibroblasts.

\section{Materials and Methods}

\section{Subjects}

Coronal suture periosteal fibroblasts and MSCs from three unrelated AS patients and from three age- and sex-matched control subjects were obtained as previously described [17]. The presence of the c.934 G>C (p.S252W) mutation was confirmed by direct DNA sequencing and expression of the mesenchyme-specific isoform of FGFR2 in the primary fibroblasts was examined by Western Blot and RT-PCR [17].

\section{Cell Culture}

Periostea harvested from AS patients or control individuals were split in half for fibroblast and MSC extraction. Primary periosteal fibroblasts derived from periosteal flaps were grown in fibroblast growth medium (DMEM High-Glucose, 20\% fetal bovine serum [FBS; GIBCO] and $100 \mathrm{U} / \mathrm{mL}$ penicillin and $100 \mu \mathrm{g} / \mathrm{mL}$ streptomycin [1\% Penicillin Streptomycin; GIBCO]). Cells were passaged at near confluency with trypsin-EDTA. MSC cultures were obtained from finely minced periosteum after $30 \mathrm{~min}$ of trypsin incubation and grown in MSC growth medium (DMEM-F12 [Invitrogen] supplemented with $15 \%$ FBS [GIBCO] and 1\% Penicillin Streptomycin [GIBCO]). All cells were cultured in a humidified incubator at $37^{\circ} \mathrm{C}$ and $5 \% \mathrm{CO}_{2}$. All tests were performed between the third and the fifth subcultures. 
For each of the 12 cell lines (three S252W fibroblasts, three WT fibroblasts, three S252W MSCs and three WT MSCs), we performed experiments in technical triplicates. For all the experiments, we used all twelve cell lines for each condition, the exceptions are indicated by an "n" value. Thus we tried to ensure that the results we obtained were representative of the biological variance seen in human patients.

\section{Immunophenotyping}

To analyze cell-surface expression of typical protein markers, adherent cells were incubated with the following anti-human primary antibodies: CD29-PECy5, CD31-phycoerythrin (PE), CD45-fluorescein isothiocyanate (FITC), CD90-R-PE, CD117-PE, and SH3 (Becton Dickinson). A total of 5,000 labeled cells were analyzed using a Guava EasyCyte flow Cytometer running Guava ExpressPlus software (Guava Technologies).

\section{Cell Proliferation Analysis}

A density of 4,000 cells $/ \mathrm{cm}^{2}$ was plated to each well of a 12-well flat bottom plate in fibroblast growth medium. After $24 \mathrm{~h}$, when total cell adhesion was verified, the fibroblasts were serum-starved for $24 \mathrm{~h}$ and MSCs for $48 \mathrm{~h}$. At the initial time point $(0 \mathrm{~h})$, we changed the starvation medium (fibroblast growth medium or MSC growth medium without FBS) for the respective cell growth medium or starvation medium supplemented with $0.5 \%$, $10 \%$ and $20 \%$ FBS. At the indicated times, the cells were trypsinized and counted using Guava EasyCyte Flow Cytometer (Guava Technologies). The experiment was done in triplicates for each time point and cell line.

\section{In Vitro Wound Healing Assay}

We plated the cells $\left(3 \times 10^{5}\right)$ on 12-well culture plates (Corning) in the respective cell growth medium. Upon reaching $100 \%$ confluence, the fibroblasts were serumstarved for another $24 \mathrm{~h}$ and MSCs for $48 \mathrm{~h}$. After starvation, a single wound was created in the center of the cell monolayer by gentle removal of the attached cells with a sterile plastic pipette tip. The cell layer was then scratched with a P-200 pipette tip, the debris was removed by washing with PBS (Phosphate Buffered Saline) and we added fibroblast or MSC growth medium. Photographs of the wound adjacent to reference lines scraped on the bottom of the plate were taken using an Axio Observer microscope under $5 \times$ field (Zeiss) at $0 \mathrm{~h}$ and $12 \mathrm{~h}$ after the wound was done. We used the ImageJ software [33] and Adobe Photoshop CS3 (Adobe) to analyze and calculate the number of cells that moved into the wound. The experiment was done in triplicates for each treatment and each cell line.

\section{In Vitro Osteogenic Differentiation}

To induce osteogenic differentiation, periosteal fibroblasts and MSCs from three AS patients and from three controls were plated in 24-well plates $\left(5 \times 10^{3} \mathrm{cells} / \mathrm{cm}^{2}\right)$ and cultured for 3 weeks in osteogenic medium (DMEM Low-Glucose, $0.5 \%$ FBS [GIBCO], $0.1 \mathrm{mM}$ dexamethasone (Sigma-Aldrich Corp., St. Louis, MO), $50 \mathrm{mM}$ ascorbate-2-phosphate (Sigma-Aldrich), $10 \mathrm{mM} \beta$-glycerophosphate (SigmaAldrich), and $1 \%$ Penicillin Streptomycin [GIBCO]). For the co-culture assay, the cells were plated at the same concentration onto 12-mm transwell inserts of 12-well plates, $0.4 \mu \mathrm{m}$ pore size (Corning Costar). Media changes occurred every three to 4 days. As an internal control of the experiment, the same cells were maintained throughout the 21 days of differentiation in regular growth medium.

Alkaline phosphatase activity was assessed on the 9th day of differentiation through a biochemical assay. The cells were provided with phosphatase substrate (SigmaAldrich) and the re-sulting p-nitrophenol was measured colorimetrically by the use of a Multiskan EX ELISA plate reader (Thermo Scientific) at $405 \mathrm{~nm}$.

After 14 and 21 days, calcified matrix production was analyzed by alizarin red staining and quantification was done as previously described [34].

\section{In Vivo Osteogenic Differentiation}

A $4.5 \mathrm{~mm}$ in diameter ceramic scaffold $(60 \%$ hydroxyapatite and $40 \%$ of $\beta$-tricalcium phosphate; Cellceram Scaffdex ${ }^{\mathrm{TM}}$ ) was moistened with osteogenic medium and mixed with $10^{6}$ human fibroblasts or MSCs. The cells attached to the scaffold were pre-differentiated in osteogenic medium and incubated at $37^{\circ} \mathrm{C}$ in $5 \% \mathrm{CO}_{2}$ for 5 days.

For the in vivo differentiation we used 8 nonimmunosuppressed (NIS) Wistar rats (all males, aged 2 months, weighing a maximum of $200 \mathrm{~g}$ ) as previously described by our group and approved by the ethical committee of our Institute [35]. We used a trephine bur of $4.5 \mathrm{~mm}$ diameter to obtain two cranial critical defects which were made in the parietal region, lateral to the sagittal suture, where two scaffolds were implanted per animal, one side being filled by biomaterial alone (left defect) and the other by the biomaterial associated with cells (right defect). The animals were kept in ventilated racks with standard conditions of temperature and lighting $\left(22^{\circ} \mathrm{C}, 12 \mathrm{~h}\right.$ light cycling per day) with free access to food and water. Four weeks after surgery, the rats were sacrificed in a $\mathrm{CO}_{2}$ chamber. The calvaria was removed and fixed in $10 \%$ formalin for $24 \mathrm{~h}$ and then decalcified in $5 \%$ formic acid for $48 \mathrm{~h}$ and embedded in paraffin. Slices of $5 \mu \mathrm{m}$ were obtained and stained with hematoxylin and eosin. 
We analyzed three transversal $4 \mu \mathrm{m}$ slices of the calvaria with $10 \mu \mathrm{m}$ of distance of each animal. Ossification area of each defect was calculated through Axio Vision Carl Zeiss based on 10x amplified images obtained from Axio Observer.A1 Carl Zeiss microscope. The percentage of the defect area that ossified at the right side was normalized by the percentage of the defect area that ossified at the left side, so that for each animal we obtained 3 ratio values.

\section{JNK Inhibitor Treatment}

To test for the role of JNK in the enhanced osteogenic potential of S252W fibroblasts, we used the reversible ATPcompetitive JNK inhibitor SP600125 (Sigma-Aldrich). Stock solutions of at least $20 \mathrm{mM}$ were made using $100 \%$ dimethyl sulfoxide.

First, we evaluated the levels of p-JNK to confirm the inhibition of JNK activity by SP600125, since the inhibitor has been described to downregulate the autophosphorilation activity of JNK [36]. Total protein extracts were prepared using Phosphosafe extraction reagent (EMD Biosciences) according to the protocol provided by the manufacturer. The levels of P-JNK were assessed by western blotting. The antibodies used were anti-p-JNK (Thr183/Tyr185) Rabbit mAb (Cell Signaling), anti-SAPK/JNK Rabbit mAb (Cell signaling), antiRabbit IgG-HRP (Cell Signaling) and Anti-B-actin-HRP (AbCam) (Supplementary figure 1).

During osteogenic differentiation, media were supplemented with $2 \mu \mathrm{M}$ and $4 \mu \mathrm{M}$ of the inhibitor, which corresponds to IC50 and twice the IC50 respectively [37].

\section{Statistical Analysis}

Continuous variables were expressed by mean and standard deviation, and the groups were compared by Student's $t$-test. A $p$ value $<0.05$ was considered statistically significant. The tests were performed using the GraphPad InStat software (GraphPad).

\section{Results}

Characterization of the Immunophenotype

In order to certify that the results we obtained were representative of the biological variance seen in human patients, we performed all experiments in fibroblast or MSC cultures from three unrelated AS patients (S252W cells) and from three control individuals (WT cells). Each cell culture was studied in three technical replicates.

All cell cultures were adherent and with a fibroblast-like appearance. We performed flow cytometry experiments with different markers in order to characterize the immunophenotype of fibroblast and MSC cultures (Table 1). S252W and WT cells were highly positive for mesenchymal cell markers $(>85 \%)$ and negative for hematopoietic and endothelial cell markers. These results confirm that these cells are of mesenchymal origin.

\section{Cell Proliferation and Cell Migration}

In order to determine the effect of the FGFR2 $2^{\mathrm{S} 252 \mathrm{~W}}$ mutation in periosteal cells, we first accessed whether the presence of the mutation altered the proliferation rates of S252W fibroblasts and S252W MSCs. The S252W mutation increased cell proliferation in fibroblasts at all times of culture (24 h: $p<0.001,48 \mathrm{~h}: p<0.001 ; 72 \mathrm{~h}: p<$ $0.001)$ (Fig. 1a) and in different culture conditions $(0.5 \%$ FBS medium: $p=0.014 ; 10 \%$ FBS medium: $p=0.04$; and $20 \%$ FBS medium: $p<0.001$ ) (Fig. 1c). On the other hand, in MSCs, the mutation decreased cell proliferation after $72 \mathrm{~h}$ in MSC growth medium (72 h: $p=0.002)$ (Fig. 1b) and in enriched medium (20\% FBS medium: $p=0.004)$ (Fig. 1d).

We next evaluated the effect of FGFR2 $2^{\mathrm{S} 252 \mathrm{~W}}$ on cell migration. The $\mathrm{S} 252 \mathrm{~W}$ mutation increased cell migration in fibroblasts only in restrictive medium condition $(0.5 \% \mathrm{FBS}$ medium: $p<0.001$ ) (Fig. 1e), but had no effect in MSCs (Fig. 1f).
Table 1 Percentage of positive cells for mesenchymal (SH3, CD90, CD29), hematopoietic (CD117 and CD45) and endothelial (CD31) cell lines antibodies

\begin{tabular}{|c|c|c|c|c|}
\hline & \multicolumn{2}{|l|}{ MSCs } & \multicolumn{2}{|l|}{ Fibroblasts } \\
\hline & $\mathrm{S} 252 \mathrm{~W}(n=2)$ & $\mathrm{WT}(n=2)$ & $\mathrm{S} 252 \mathrm{~W}(n=4)$ & $\mathrm{WT}(n=4)$ \\
\hline $\mathrm{SH} 3$ & $96,32-99,78 \%$ & $94,7-98,68 \%$ & $88,92-98,45 \%$ & $93,92-97,87 \%$ \\
\hline CD90 & $94,92-95,52 \%$ & $94,02-98,7 \%$ & $89,12-99,7 \%$ & $89,46-98,94 \%$ \\
\hline $\mathrm{CD} 29$ & $98,48-99,12 \%$ & $88,1-98,98 \%$ & $85,14-99,02 \%$ & $70-99,25 \%$ \\
\hline CD31 & $<5 \%$ & $<5 \%$ & $<5 \%$ & $<5 \%$ \\
\hline CD117 & $<5 \%$ & $<5 \%$ & $<5 \%$ & $<5 \%$ \\
\hline CD45 & $<5 \%$ & $<5 \%$ & $<5 \%$ & $<5 \%$ \\
\hline
\end{tabular}


Fig. 1 a Comparative analysis of the proliferation of WT (from 3 individuals) and S252W (from 3 patients) fibroblasts and b WT (from 3 individuals) and S252W (from 3 patients) MSCs. Each point indicates the average for each time and each condition and the error bars represent the standard deviation for the biological replicate (same S252W or WT cell type culture results summed). Analysis of c WT (from 3 individuals) and S252W (from 3 patients) fibroblasts and $\mathbf{d}$ WT (from 3 individuals) and S252W (from 3 patients) MSCs when grown in culture medium with different FBS concentration. Each point indicates the average of each medium after $48 \mathrm{~h}$ and the error bars represent the standard deviation for the biological replicate. e Wound healing assay of S252W $(n=3)$ and WT $(n=3)$ fibroblasts and $\mathbf{f}$ and S252W $(n=3)$ and WT $(n=3)$ MSCs in high FBS and low FBS growth medium. The bars represent the average number of cells that migrated toward the wound after $12 \mathrm{~h}$ for each condition and error bars represent the standard deviation for the biological replicate $(*: p<0.05, * *$ : $p<0.01, * * *: p<0.001)$
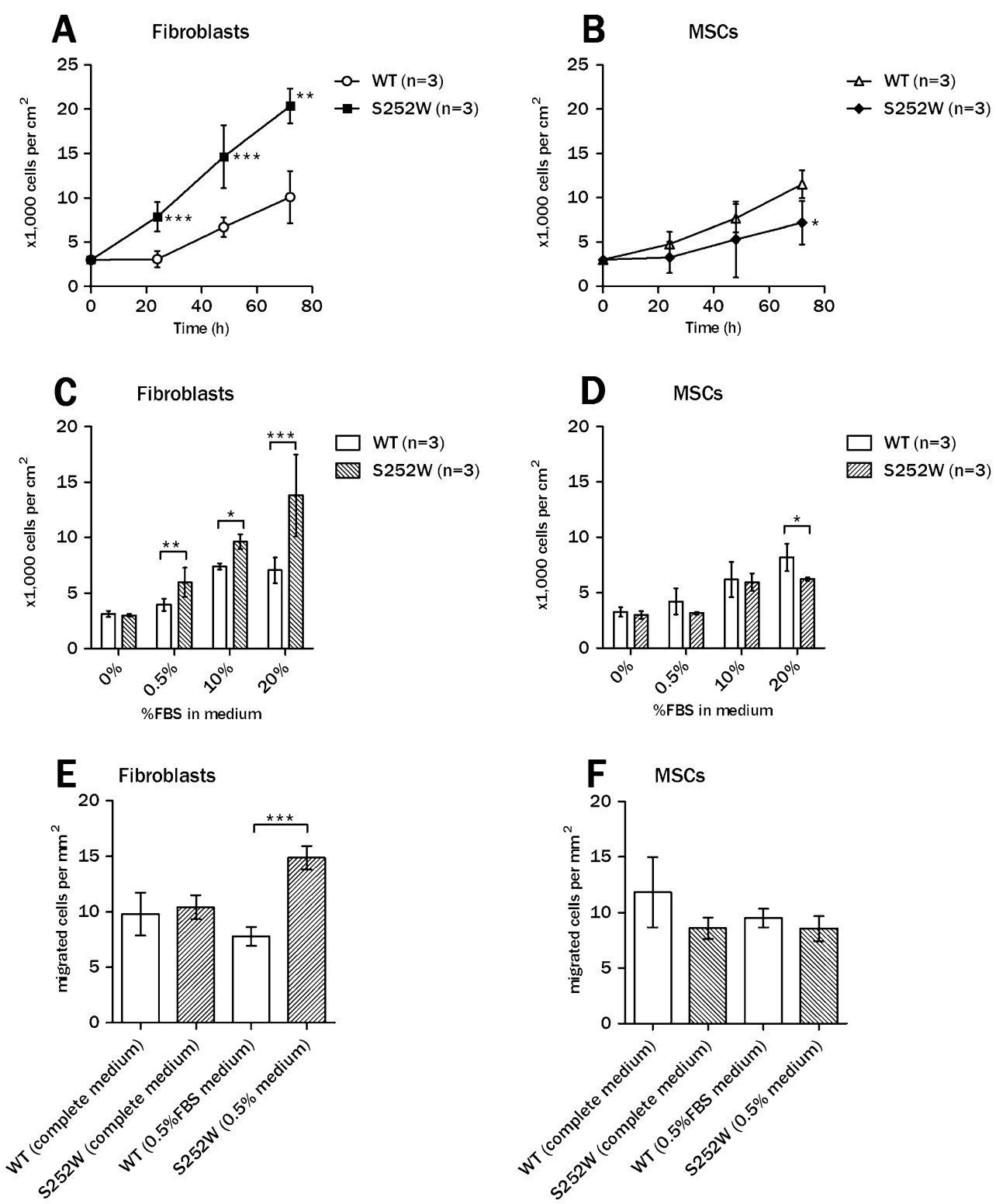

In Vitro Osteogenic Differentiation

Next, we assessed the effects of the S252W mutation on osteogenic differentiation. We performed experiments at key points of our in vitro differentiation protocol in order to evaluate whether the mutation plays a more prominent part at a specific stage of osteogenic differentiation.

The ninth day of differentiation is ideal to access the levels of alkaline phosphatase (ALP) in cultures, as in this period there is a peak production of the enzyme. ALP provides the phosphate needed for the production of cellular matrix calcium. S252W fibroblasts showed 6-fold increase in ALP activity in comparison to WT fibroblasts $(p<0.001)$ (Fig. 2a), while S252W MSCs had 3-fold increase in comparison to WT MSCs $(p<0.001)$ (Fig. 2b).

After 2 weeks in osteogenic medium, we analyzed initial calcium deposition in the extracellular matrix (ECM) through alizarin red staining. S252W fibroblasts showed 2.7-fold increase in ECM calcium in comparison to WT fibroblasts $(p<0.001)$ (Fig. 2c), while S252W MSCs had 1.5 -fold increase in comparison to WT MSCs $(p=0.016)$ (Fig. 2d).

Finally, we evaluated concentration of ECM calcium at the end of differentiation (21st day). S252W fibroblasts showed a 1.7-fold increase in ECM calcium in comparison to WT fibroblasts $(p=0.002)$ (Fig. 2e), while S252W MSCs had a 1.5 -fold increase in comparison to WT MSCs $(p<$ 0.001) (Fig. 2f).

\section{In Vivo Osteogenic Differentiation}

To validate these data in vivo, we performed the bilateral cranial critical defect experiment using Wistar NIS rats as previously described by our group [35]. At the right-side 
defect we introduced either S252W or WT cells associated with biomaterial, and at the left-side defect we introduced biomaterial only as an internal control of each animal's osteoregeneration. Four weeks after the surgery, the rightside:left-side ossification ratio was 4.9 in S252W fibroblasts and 1.9 compared to WT fibroblasts (2.6-fold higher; $p=$ 0.036) (Fig. 2g). Likewise, this ratio was 11.8 in S252W MSCs and 2.6 in WT MSCs (4.5-fold higher; $p=0.001$ ) (Fig. 2h).

Interactions between Periosteal MSCs and Fibroblasts During Osteogenic Differentiation

As shown above, we observed in vitro and in vivo that the cellular phenotype alterations due to S252W mutation seemed more drastic in fibroblasts than in MSCs, and that S252W fibroblasts are particularly more prone to osteogenic differentiation. These data thus raised the question whether S252W fibroblasts could induce osteogenic differentiation in other cells.

Therefore, in order to test the hypothesis that a cell population with the S252W mutation alters normal signaling in adjacent cells, we used a co-culture system to simulate the in vivo anatomic link between the fibroblasts and MSCs in the periosteum, allowing the paracrine signaling without physical cell interaction. S252W fibroblasts induced $30 \%$ more differentiation of periosteal MSCs, whether WT $(n=3)$ or S252W $(n=2)$, both by ALP assay (WT MSCs: $p<0.001$; S252W MSCs: $p=0.037$ ) and alizarin red staining (vs. WT MSCs: $p=0.007$; vs. S252W MSCs: $p=0.016$ ) (Fig. $3 \mathrm{a}$ and c). Interestingly, S252W fibroblasts did not induce osteogenic differentiation of MSC from another tissue, such as dental pulp stem cells (DPSC) (Fig. 3a and c). Further, S252W MSCs and WT MSCs exhibit no influence on the osteogenic differentiation of S252W fibroblasts (Fig. 3b and d).

\section{Potential Molecule Involved in Altered Fibroblast Osteogenic Potential}

To assess whether JNK plays a role in the increased osteogenic potential of S252W fibroblasts, we treated S252W fibroblasts with $2 \mu \mathrm{M}$ (IC50) [37] and $4 \mu \mathrm{M}$ (twice IC50) of SP600125, a JNK phosphorylation inhibitor, during osteogenic differentiation. We observed a lower ALP activity as we increased the concentration of SP600125 (untreated vs. $+2 \mu \mathrm{M}$ SP600125: $p=0.025$; $+2 \mu \mathrm{M}$ SP600125 vs. $+4 \mu \mathrm{M}$ SP600125: $p=0.014)$. This effect was also observed by alizarin red staining (untreated vs. $+2 \mu \mathrm{M}$ SP600125: $p=0.006$; untreated vs. $+4 \mu \mathrm{M}$ SP600125: $p=0.003$ ) (Fig. $4 \mathrm{a}$ and b). At the maximal inhibition of JNK (4 $\mu \mathrm{M})$, ALP activity of S252W fibroblast and WT fibroblasts were equivalent. Therefore,
Fig. 2 S252W and WT fibroblasts and MSCs (all conditions: $n=3$ ) in response to osteogenic medium during different phases of osteogenic differentiation. a, b Analysis of alkaline phosphatase activity on the 9th day of osteogenic differentiation; c, d alizarin red staining quantification at the 14th day of differentiation; e, f alizarin red staining quantification at the 21 st day of osteogenic differentiation. The columns represent the absorbance at wavelength indicated for each condition and error bars represent the standard deviation for the biological replicate. g Percentage of ossification area of calvarial defects with WT or S252W fibroblasts in rats 4 weeks after surgery. h Percentage of ossification area of calvarial defects with WT or S252W MSCs in rats 4 weeks after surgery $(*: p<0.05$; **: $p<0.01$; $* * *: p<0.001)$

inhibition of JNK activity rescued the increased osteogenic potential of S252W fibroblasts.

\section{Discussion}

The only treatment available nowadays for AS patients is surgical intervention, which consists of artificial reconstruction of the coronal sutures [38]. Nevertheless, postsurgical ossification of the sutures (resynostosis) is frequent and any surgical treatment for craniosynostosis during childhood is considered a procedure to delay but not to prevent synostosis [1]. To understand the cause and prevent resynostosis of the sutures so that they remain open during the growth phase of the individual, it is important to identify the main molecules and mechanisms involved in this process. This knowledge can contribute to a better management of the affected child, enhancing their life quality. Bone regeneration studies have pointed to the periosteum as a significant contributor to the ossification process $[22,23]$, not only through molecular signaling, but also by providing osteoprogenitor cells [26]. Despite the periosteum's possible contribution to premature suture ossification in AS and other craniosynostosis, the mechanism by which FGFR2 gain-of-function mutations achieve this effect is not clear. Unbalanced FGF signaling can accelerate proliferation, migration, and differentiation in osteoprogenitor cells from the periosteum and contribute to resynostosis, though this process continues to be poorly understood, particularly in AS patients. Study of cells derived from the periosteum of the AS patients can help to elucidate these questions, as we can compare the effect of the mutation in different cell types from the same niche. This could be of particular relevance considering that cell signaling can be different between mice and humans, and that the range of phenotypic effects of a mutation is not always the same in both [13-16]. Here we successfully established fibroblasts and MSCs cell cultures from the periosteum of both AS and control individuals. In accordance to the literature [38], we were not able to distinguish these two cell types based on morphology and positive staining for mesenchymal cell markers. However, the 

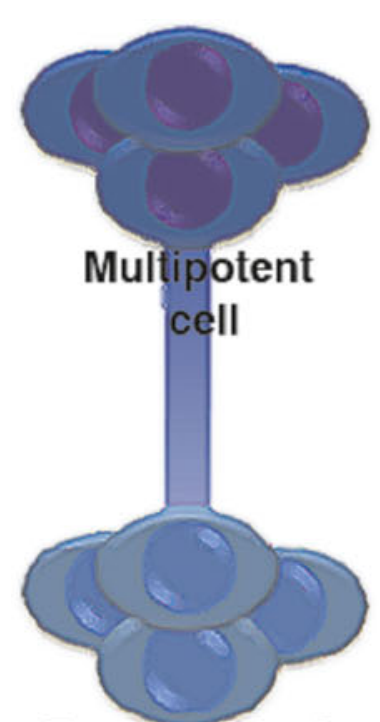

Preosteoblasts
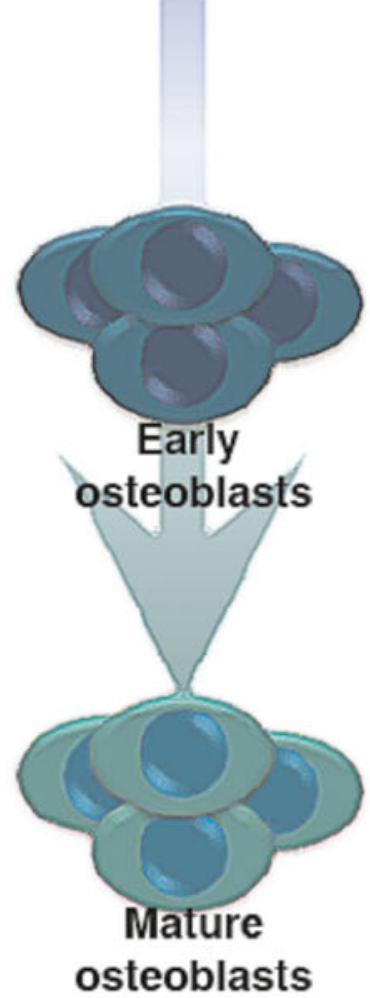

A Day 9: Fibroblasts

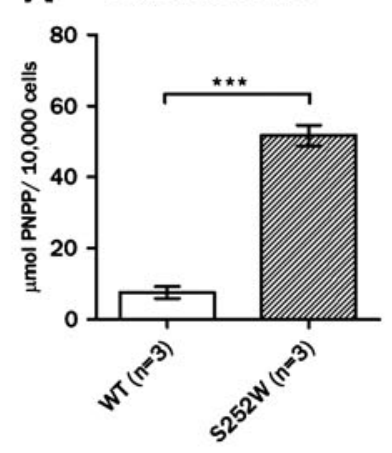

C Day 14: Fibroblasts

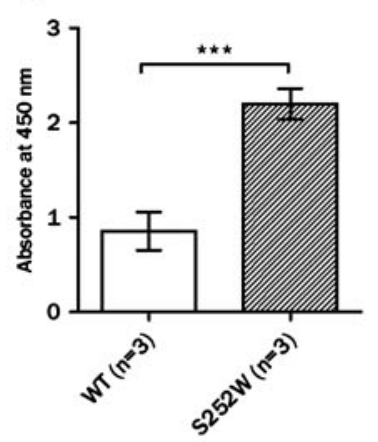

E Day 21: Fibroblasts

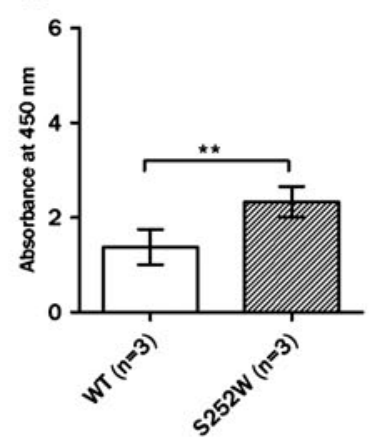

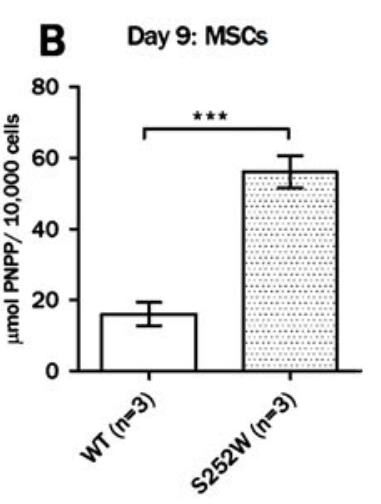

D Day 14: MSCs

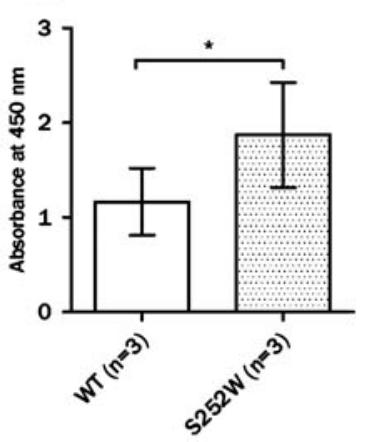

F Day 21: MSCs

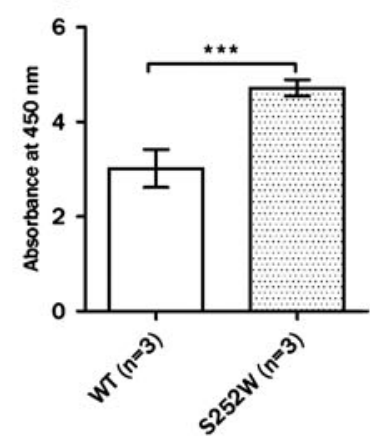

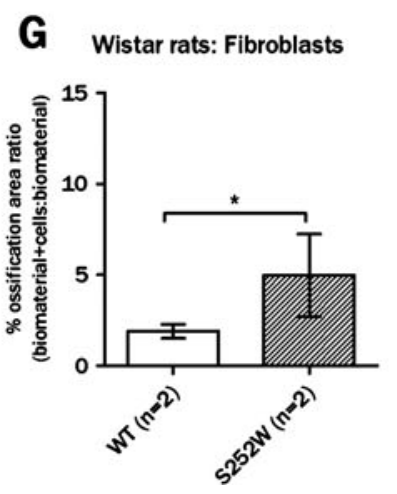

H Wistar rats: MSCs

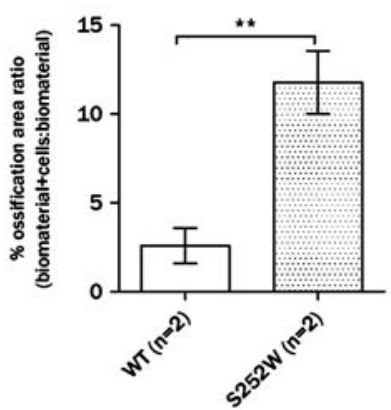


Fig. 3 Effects of interaction between periosteal MSCs and fibroblasts. a Analysis of alkaline phosphatase activity on the 9th day and $\mathbf{c}$ alizarin red staining at the 21st day of osteogenic differentiation of S252W MSCs $(n=2)$, WT MSCs $(n=2)$ and WT DPSC $(n=1)$ co-cultured with fibroblasts with or without the mutation in the presence of osteogenic medium. b Analysis of alkaline phosphatase activity on the 9th day and $\mathbf{d}$ alizarin red staining at the 21 st day of osteogenic differentiation of S252W fibroblast $(n=1)$ cocultured with MSCs with or without the mutation in the presence of osteogenic medium. The columns represent the absorbance at wavelength indicated for each condition and error bars represent the standard deviation for the biological replicate $(*: p<0.05, * *$ : $p<0.01, * * *: p<0.001)$
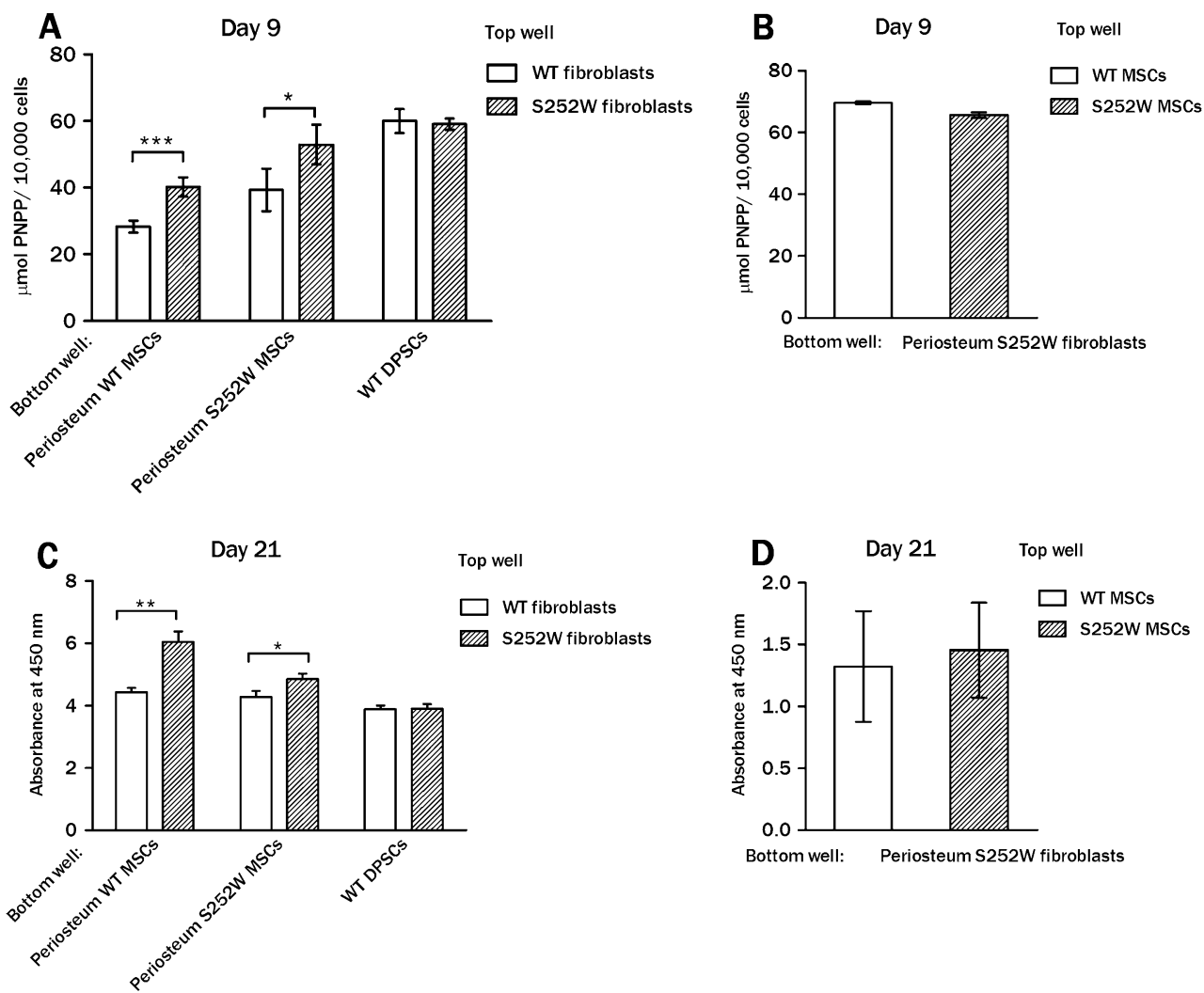

positive bone differentiation seen in WT MSCs but not in WT fibroblasts confirms that our protocols allow for the establishment of two distinct cell populations, as we have shown in our previous reports [17, 39].

The S252W mutation enhanced cell proliferation in fibroblasts, even in critical culture conditions, such as $0.5 \%$ FBS medium, but it had a negative effect on MSCs in $20 \%$ FBS culture environment. Literature data regarding the presence of FGFR $2^{\mathrm{S} 252 \mathrm{~W}}$ and cell proliferation are controversial. Some studies that investigated human periosteal fibroblasts [17], murine osteoblasts [8], murine stem cells [40], or calvarial cells during embryonic sutures formation in murine AS models [41, 42] point to the FGFR2 $2^{\mathrm{S} 252 \mathrm{~W}}$ mutation as responsible for increasing cell proliferation. Interestingly, the murine MSCs with FGFR3 mutation proliferate less than the wild type cells [43]. These comparative analyses are complex, as the experiments have not only included different cell types but also cells from different species and from different niches. Therefore, although we cannot rule out differences in the protocols used, our results suggest that the effect of the FGFR2 $2^{\mathrm{S} 252 \mathrm{~W}}$ mutation on cell proliferation might depend both on the tissue, niche of origin, and cell type under
Fig. 4 Effects of JNK inhibition in periosteal $\mathrm{S} 252 \mathrm{~W}(n=2)$ and WT $(n=2)$ fibroblasts. a Analysis of alkaline phosphatase activity on the 9th day of osteogenic differentiation. b Alizarin red staining quantification at the 21 st day of osteogenic differentiation. The columns represent the absorbance at wavelength indicated for each condition and error bars represent the standard deviation for the biological replicate $(*: p<0.05, * *$ : $p<0.01, * * *: p<0.001)$
Day 9
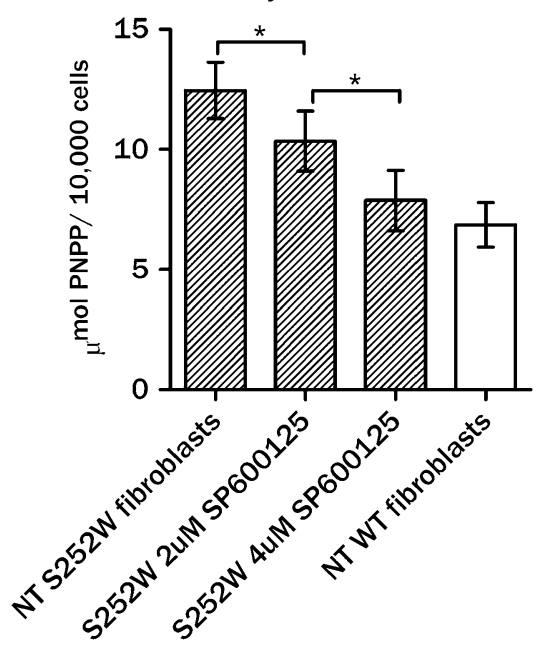

Day 21

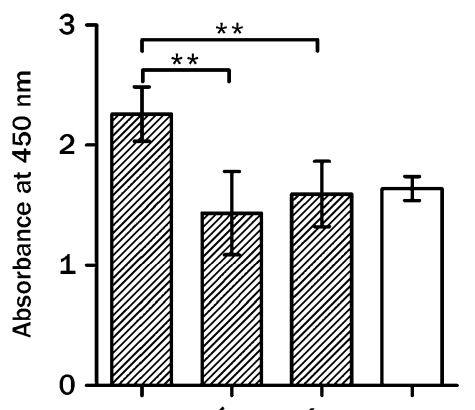

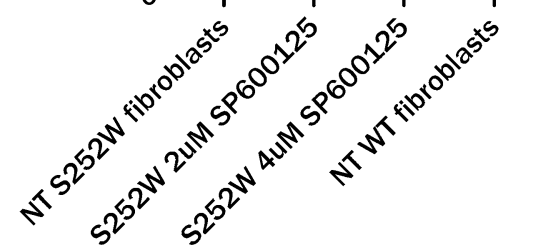


Fig. 5 In the periosteum (pink) overlying the suture (brown), fibroblasts (green cells) and MSCs (blue cells) have similar cell growth and cell migration rates. However, fibroblasts exhibit low while MSCs show higher osteogenic potential (left). The FGFR2 ${ }^{\mathrm{S} 252 \mathrm{~W}}$ mutation has a positive effect on both proliferation and migration of fibroblasts, and a negative effect on MSC proliferation. It has no consequence on MSCs migration. Both cell types have enhanced osteogenic differentiation and S252W fibroblasts show positive influence on MSCs differentiation. Inhibition of JNK phosphorylation by SP600125 can null the effect of the mutation over osteogenic differentiation of fibroblasts

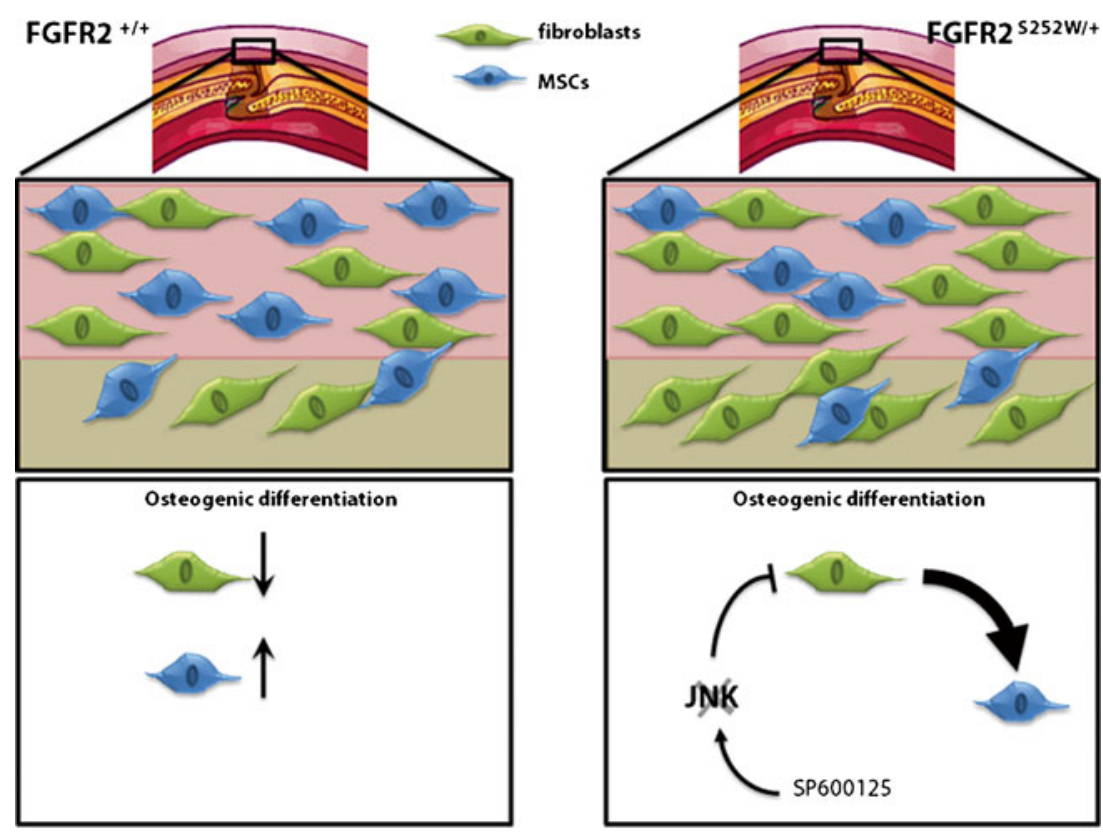

analysis, which would explain at least in part the controversy in the literature.

Through the wound healing assay, we have shown that FGFR2 ${ }^{\mathrm{S} 252 \mathrm{~W}}$ has an effect on the migratory property of fibroblasts, but not of MSCs. This effect on the fibroblasts, however, was dependent on the availability of FBS, a culture medium supplement that provides not only growth factors, but also cellular growth inhibitors [44]. It was previously reported that FGFR2 ${ }^{\mathrm{S} 252 \mathrm{~W}}$ has enhanced affinity to different FGFs [45], therefore, the altered S252W cell proliferation and cell migration in response to different FBS concentrations suggests that FBS contains growth stimulating FGFs to which S252W fibroblasts are more sensitive to, and FGFs that in high concentration inhibit migration of $\mathrm{S} 252 \mathrm{~W}$ fibroblasts. It is possible that during embryonic and even post-natal development, atypical cell responses are dependent on which FGF is available at that period.

Regarding the osteogenic potential, we found that FGFR2 ${ }^{\text {S252W }}$ induces increased osteogenic differentiation in both cell types under study, especially at the early stages of the in vitro process. We were able to confirm these results in vivo using non-immunosuppressed rats and human cells, as an increased ossification rate was observed for both fibroblasts and MSCs harboring the FGFR2 ${ }^{\mathrm{S} 252 \mathrm{~W}}$ mutation as compared to corresponding WT cells. These results thus confirm our preliminary work on S252W fibroblasts [13] which showed that the FGFR2 $2^{\mathrm{S} 252 \mathrm{~W}}$ mutation confers a new function to these cells. In addition, our results for MSCs, the osteoblast precursors, are in agreement with most of the literature, ranging from studies with murine stem cells to human pre-osteoblasts transfected with FGFR2 ${ }^{\mathrm{S} 252 \mathrm{~W}}$, which showed that the FGFR2 ${ }^{\mathrm{S} 252 \mathrm{~W}}$ mutation enhances osteogenic potential [8, 9, 40, 41, 46].
A dynamic system based on integrated signals between stem cells and cells from their surrounding niche, such as fibroblasts, is necessary to maintain proper tissue physiology [47]. Thus, we judged it necessary to evaluate the effect of S252W fibroblasts on the MSCs, the expected osteoblast precursors. Interestingly, our co-culture assay showed that the presence of $\mathrm{S} 252 \mathrm{~W}$ fibroblasts promotes osteogenic differentiation of both S252W and WT periosteal MSCs. In addition, S252W fibroblasts did not induce osteogenic differentiation in DPSC, which suggests that the cells we used must still harbor regulatory network programs that are specific to the tissue from which they are extracted and that are not erased in cell culture.

The increased osteogenic potential of S252W fibroblasts seems to be mediated by JNK, since JNK inhibition reverted this phenotype. Shukla et al. demonstrated that ERK (extracellular-signal-regulated kinase) is directly involved in the craniosynostosis in a Fgfr $2^{+/ \mathrm{S} 252 \mathrm{~W}}$ mouse [48]. Futhermore, ERK indirectly increases JNK activity [49]. Our findings thus suggest that ERK-JNK pathway is also disturbed in human AS patients and that a key molecule involved in craniosynostosis lies downstream to ERK and JNK. Altogether, we propose that identifying this key molecule might have a better therapeutic potential in the surgical treatment of AS patients than ERK and JNK inhibitors, as inhibition of either molecule leads to severe side effects as both are involved in important signaling pathway in the whole organism. We cannot rule out the possibility that other MAPKs, such as p38, downstream to FGFR activation might be responsible for the increased osteogenic potential in the $\mathrm{S} 252 \mathrm{~W}$ fibroblasts, as these molecules have been shown to have enhance activation in Apert mouse models [41, 43, 48, 50-54]. 
Based on our results, we propose that the FGFR2 ${ }^{\mathrm{S} 252 \mathrm{~W}}$ mutation confers a most pronounced gain-of-function in fibroblast cells. Of the cell phenotypes evaluated, the most strikingly altered one is their increased osteogenic potential. This represents an acquired new function for fibroblasts, apparently mediated by JNK pathway. It has been suggested that the premature suture fusion in S252W cells is a result of excessive cell proliferation [7]. In the present study, FGFR2 ${ }^{\mathrm{S} 252 \mathrm{~W}}$ mutation leads to increased proliferation, migration, and osteogenic potential of both fibroblasts and MSCs. Therefore, the premature ossification process might result from a more complex mechanism than only alteration of the proliferative capacity. Further, we show that fibroblasts enhance the osteogenic potential of MSCs of the same niche. These results allow us to suggest that periosteum cells might contribute to premature suture fusion in these patients (Fig. 5), a characteristic that has previously been attributed to dura mater [49]. To better understand the molecular mechanisms underlying our findings, it is crucial to identify molecules secreted by S252W fibroblasts that may contribute to intensify osteogenic differentiation in other cells and whether they are related to the JNK pathway. These molecules could lead to the identification of candidate drugs that could ameliorate the surgical prognosis of AS patients.

Disclosures The authors indicate no potential conflicts of interest.

Open Access This article is distributed under the terms of the Creative Commons Attribution Noncommercial License which permits any noncommercial use, distribution, and reproduction in any medium, provided the original author(s) and source are credited.

\section{References}

1. Cohen, M. M. J. M. (2000). R. E. Craniosynostosis: Diagnosis, evaluation and manegement (2nd ed.). USA: Oxford University Press.

2. Cohen, M. M., Jr., \& Kreiborg, S. (1993). Skeletal abnormalities in the Apert syndrome. American Journal of Medical Genetics, 47 (5), 624-632.

3. Murnaghan, L. M., Thurgur, C. H., Forster, B. B., Sawatzky, B. J., Hawkins, R., \& Tredwell, S. J. (2007). A clinicoradiologic study of the shoulder in Apert syndrome. Journal of Pediatric Orthopedics, 27(7), 838-843.

4. McHugh, T., Wyers, M., \& King, E. (2007). MRI characterization of the glenohumeral joint in Apert syndrome. Pediatric Radiology, 37(6), 596-599.

5. Ornitz, D. M. (2000). FGFs, heparan sulfate and FGFRs: complex interactions essential for development. Bioessays, 22 (2), 108-112.

6. Ibrahimi, O. A., Eliseenkova, A. V., Plotnikov, A. N., Yu, K., Ornitz, D. M., \& Mohammadi, M. (2001). Structural basis for fibroblast growth factor receptor 2 activation in Apert syndrome.
Proceedings of the National Academy of Sciences of the United States of America, 98(13), 7182-7187.

7. Beenken, A. O. (2011) L.A.

8. Yang, F., Wang, Y., Zhang, Z., Hsu, B., Jabs, E. W., \& Elisseeff, J. H. (2008). The study of abnormal bone development in the Apert syndrome Fgfr2+/S252W mouse using a 3D hydrogel culture model. Bone, 43(1), 55-63.

9. Lomri, A., Lemonnier, J., Hott, M., et al. (1998). Increased calvaria cell differentiation and bone matrix formation induced by fibroblast growth factor receptor 2 mutations in Apert syndrome. The Journal of Clinical Investigation, 101(6), 1310 1317.

10. Mansukhani, A., Bellosta, P., Sahni, M., \& Basilico, C. (2000). Signaling by fibroblast growth factors (FGF) and fibroblast growth factor receptor 2 (FGFR2)-activating mutations blocks mineralization and induces apoptosis in osteoblasts. The Journal of Cell Biology, 149(6), 1297-1308.

11. Mestas, J., \& Hughes, C. C. (2004). Of mice and not men: differences between mouse and human immunology. Journal of Immunology, 172(5), 2731-2738.

12. Haley, P. J. (2003). Species differences in the structure and function of the immune system. Toxicology, 188(1), 49-71.

13. Bulfield, G., Siller, W. G., Wight, P. A., \& Moore, K. J. (1984). X chromosome-linked muscular dystrophy $(\mathrm{mdx})$ in the mouse. Proceedings of the National Academy of Sciences of the United States of America, 81(4), 1189-1192.

14. Dhillon, K. K., Sidorova, J. M., Albertson, T. M., et al. (2009). Divergent cellular phenotypes of human and mouse cells lacking the Werner syndrome RecQ helicase. DNA Repair, 9(1), 11-22.

15. Harries, L. W., Brown, J. E., \& Gloyn, A. L. (2009). Species-specific differences in the expression of the HNF1A, HNF1B and HNF4A genes. PloS one, 4(11), e7855.

16. Twigg, S. R., Healy, C., Babbs, C., et al. (2009). Skeletal analysis of the Fgfr3(P244R) mouse, a genetic model for the Muenke craniosynostosis syndrome. Developmental Dynamics, 238(2), 331-342.

17. Fanganiello, R. D., Sertie, A. L., Reis, E. M., et al. (2007). Apert p.Ser252Trp mutation in FGFR2 alters osteogenic potential and gene expression of cranial periosteal cells. Molecular Medicine (Cambridge, Mass), 13(7-8), 422-442.

18. Slater, B. J., Lenton, K. A., Kwan, M. D., Gupta, D. M., Wan, D. C., \& Longaker, M. T. (2008). Cranial sutures: a brief review. Plastic and Reconstructive Surgery, 121(4), 170e-178e.

19. Foster, K. A., Frim, D. M., \& McKinnon, M. (2008). Recurrence of synostosis following surgical repair of craniosynostosis. Plastic and Reconstructive Surgery, 121(3), 70e-76e.

20. Hopper, R. A., Zhang, J. R., Fourasier, V. L., et al. (2001). Effect of isolation of periosteum and dura on the healing of rabbit calvarial inlay bone grafts. Plastic and Reconstructive Surgery, 107(2), 454-462.

21. Ozerdem, O. R., Anlatici, R., Bahar, T., et al. (2003). Roles of periosteum, dura, and adjacent bone on healing of cranial osteonecrosis. The Journal of Craniofacial Surgery, 14(3), 371379. discussion 80-2.

22. Ito, Y., Sanyal, A., Fitzsimmons, J. S., Mello, M. A., \& O'Driscoll, S. W. (2001). Histomorphological and proliferative characterization of developing periosteal neochondrocytes in vitro. Journal of Orthopaedic Research, 19(3), 405-413.

23. Orwoll, E. (2006). Genetic aspects of osteoporosis. Annales d'endocrinologie, 67(2), 113.

24. Allen, M. R., Hock, J. M., \& Burr, D. B. (2004). Periosteum: biology, regulation, and response to osteoporosis therapies. Bone, 35(5), 1003-1012.

25. Squier, C. A., Ghoneim, S., \& Kremenak, C. R. (1990). Ultrastructure of the periosteum from membrane bone. Journal of Anatomy, 171, 233-239. 
26. Colnot, C. (2009). Skeletal cell fate decisions within periosteum and bone marrow during bone regeneration. Journal of Bone and Mineral Research, 24(2), 274-282.

27. Bullaughey, K., Chavarria, C. I., Coop, G., \& Gilad, Y. (2009). Expression quantitative trait loci detected in cell lines are often present in primary tissues. Human Molecular Genetics, 18(22), 4296-4303.

28. Gallea, S., Lallemand, F., Atfi, A., et al. (2001). Activation of mitogen-activated protein kinase cascades is involved in regulation of bone morphogenetic protein-2-induced osteoblast differentiation in pluripotent $\mathrm{C} 2 \mathrm{C} 12$ cells. Bone, 28(5), 491-498.

29. Liu, H., Liu, Y., Viggeswarapu, M., Zheng, Z., Titus, L., \& Boden, S. D. (2010). Activation of c-Jun NH(2)-terminal kinase 1 increases cellular responsiveness to BMP-2 and decreases binding of inhibitory Smad6 to the type I BMP receptor. Journal of Bone and Mineral Research

30. Matsuguchi, T., Chiba, N., Bandow, K., Kakimoto, K., Masuda, A., \& Ohnishi, T. (2009). JNK activity is essential for Atf4 expression and late-stage osteoblast differentiation. Journal of Bone and Mineral Research, 24(3), 398-410.

31. Guicheux, J., Lemonnier, J., Ghayor, C., Suzuki, A., Palmer, G., \& Caverzasio, J. (2003). Activation of p38 mitogen-activated protein kinase and c-Jun-NH2-terminal kinase by BMP-2 and their implication in the stimulation of osteoblastic cell differentiation. Journal of Bone and Mineral Research, 18(11), 2060-2068.

32. Li, C., Scott, D. A., Hatch, E., Tian, X., \& Mansour, S. L. (2007). Dusp6 (Mkp3) is a negative feedback regulator of FGF-stimulated ERK signaling during mouse development. Development (Cambridge, England), 134(1), 167-176.

33. ImageJ. U. S. National Institutes of Health, 1997-2009. (Accessed 5 October 2009, at http://rsb.info.nih.gov/ij/).

34. Gregory, C. A., Gunn, W. G., Peister, A., \& Prockop, D. J. (2004). An Alizarin red-based assay of mineralization by adherent cells in culture: comparison with cetylpyridinium chloride extraction. Analytical Biochemistry, 329(1), 77-84.

35. de Mendonca Costa, A., Bueno, D. F., Martins, M. T., et al. (2008). Reconstruction of large cranial defects in nonimmunosuppressed experimental design with human dental pulp stem cells. The Journal of Craniofacial Surgery, 19(1), 204-210.

36. Bennett, B. L., Sasaki, D. T., Murray, B. W., et al. (2001). SP600125, an anthrapyrazolone inhibitor of Jun N-terminal kinase. Proceedings of the National Academy of Sciences of the United States of America, 98(24), 13681-13686.

37. Joiakim, A., Mathieu, P. A., Palermo, C., Gasiewicz, T. A., \& Reiners, J. J., Jr. (2003). The Jun N-terminal kinase inhibitor SP600125 is a ligand and antagonist of the aryl hydrocarbon receptor. Drug Metabolism and Disposition: The Biological Fate of Chemicals, 31(11), 1279-1282.

38. Posnick, J. C., Armstrong, D., \& Bite, U. (1995). Crouzon and Apert syndromes: intracranial volume measurements before and after cranio-orbital reshaping in childhood. Plastic and Reconstructive Surgery, 96(3), 539-548.

39. Bueno, D. F., Kerkis, I., Costa, A. M., et al. (2009). New source of muscle-derived stem cells with potential for alveolar bone reconstruction in cleft lip and/or palate patients. Tissue Engineering Part A, 15(2), 427-435.

40. Miraoui, H., Oudina, K., Petite, H., Tanimoto, Y., Moriyama, K., \& Marie, P. J. (2009). Fibroblast growth factor receptor 2 promotes osteogenic differentiation in mesenchymal cells via ERK1/2 and protein kinase C signaling. The Journal of Biological Chemistry, 284(8), 4897-4904.

41. Holmes, G., Rothschild, G., Roy, U. B., Deng, C. X., Mansukhani, A., \& Basilico, C. (2009). Early onset of craniosynostosis in an Apert mouse model reveals critical features of this pathology. Developmental Biology, 328(2), 273-284.

42. Wang, Y., Xiao, R., Yang, F., et al. (2005). Abnormalities in cartilage and bone development in the Apert syndrome FGFR2(+/ S252W) mouse. Development (Cambridge, England), 132(15), 3537-3548.

43. Su, N., Sun, Q., Li, C., et al. (2010). Gain-of-function mutation in FGFR3 in mice leads to decreased bone mass by affecting both osteoblastogenesis and osteoclastogenesis. Human Molecular Genetics, 19(7), 1199-1210.

44. Freshney, R. I. (2005). Culture of animal cells: A manual of basic technique (5th ed). Wiley-Liss.

45. Ibrahimi, O. A., Zhang, F., Eliseenkova, A. V., Itoh, N., Linhardt, R. J., \& Mohammadi, M. (2004). Biochemical analysis of pathogenic ligand-dependent FGFR2 mutations suggests distinct pathophysiological mechanisms for craniofacial and limb abnormalities. Human Molecular Genetics, 13(19), 2313-2324.

46. Lemonnier, J., Hay, E., Delannoy, P., et al. (2001). Increased osteoblast apoptosis in apert craniosynostosis: role of protein kinase C and interleukin-1. The American Journal of Pathology, 158(5), 1833-1842.

47. Scadden, D. T. (2006). The stem-cell niche as an entity of action. Nature, 441(7097), 1075-1079.

48. Shukla, V., Coumoul, X., Wang, R. H., Kim, H. S., \& Deng, C. X. (2007). RNA interference and inhibition of MEK-ERK signaling prevent abnormal skeletal phenotypes in a mouse model of craniosynostosis. Nature Genetics, 39(9), 1145-1150.

49. Lopez-Bergami, P., Huang, C., Goydos, J. S., et al. (2007). Rewired ERK-JNK signaling pathways in melanoma. Cancer Cell, 11(5), 447-460.

50. Beenken, A., \& Mohammadi, M. (2009). The FGF family: biology, pathophysiology and therapy. Nature Reviews, 8(3), 235-253.

51. Ge, C., Xiao, G., Jiang, D., \& Franceschi, R. T. (2007). Critical role of the extracellular signal-regulated kinase-MAPK pathway in osteoblast differentiation and skeletal development. The Journal of Cell Biology, 176(5), 709-718.

52. Marie, P. J. (2003). Fibroblast growth factor signaling controlling osteoblast differentiation. Gene, 316, 23-32.

53. Wang, Y., Sun, M., Uhlhorn, V. L., et al. Activation of p38 MAPK pathway in the skull abnormalities of Apert syndrome Fgfr2 $(+\mathrm{P} 253 \mathrm{R})$ mice. BMC Developmental Biology, 10, 22.

54. Yin, L., Du, X., Li, C., et al. (2008). A Pro253Arg mutation in fibroblast growth factor receptor 2 (Fgfr2) causes skeleton malformation mimicking human Apert syndrome by affecting both chondrogenesis and osteogenesis. Bone, 42(4), 631-643. 